\title{
Incremental prognostic value of multi-slice computed tomography coronary angiography over coronary artery calcium scoring in patients with suspected coronary artery disease
}

\author{
Jacob M. van Werkhoven ${ }^{1,2}$, Joanne D. Schuijf', Oliver Gaemperli3,4, \\ J. Wouter Jukema ${ }^{1,2}$, Lucia J. Kroft ${ }^{5}$, Eric Boersma ${ }^{6}$, Aju Pazhenkottil ${ }^{3,4}$, \\ Ines Valenta ${ }^{4}$, Gabija Pundziute ${ }^{1}$, Albert de Roos ${ }^{5}$, Ernst E. van der Wall1,2, \\ Philipp A. Kaufmann ${ }^{4,7}$, and Jeroen J. Bax ${ }^{1 *}$
}

${ }^{1}$ Department of Cardiology, Leiden University Medical Center, Albinusdreef 2, 2333 ZA Leiden, The Netherlands; ${ }^{2}$ The Interuniversity Cardiology Institute of the Netherlands,
Utrecht, The Netherlands; ' ${ }^{3}$ Department of Cardiology, University Hospital Zurich, Zurich, Switzerland; ${ }^{4}$ Department of Nuclear Medicine, University Hospital Zurich, Zurich,
Switzerland; ${ }^{5}$ Department of Radiology, Leiden University Medical Center, Leiden, The Netherlands; ${ }^{6}$ Department of Cardiology, Erasmus Medical Center, Rotterdam,
The Netherlands; and ${ }^{7}$ Zurich Integrative Human Physiology, University of Zurich, Zurich, Switzerland

Received 26 November 2008; revised 25 May 2009; accepted 11 June 2009; online publish-ahead-of-print 29 June 2009

Aims

The purpose of this study was to assess the relationship between calcium scoring (CS) and multi-slice computed tomography coronary angiography (MSCTA) and to determine if MSCTA has an incremental prognostic value to CS.

Methods

and results

In 432 patients (59\% male, age $58 \pm 11$ years) referred for cardiac evaluation owing to suspected coronary artery disease (CAD), CS and 64-slice MSCTA were performed. The following events were combined in a composite endpoint: all-cause mortality, non-fatal infarction, and unstable angina requiring revascularization. CS was 0 in 147 (34\%) patients, CS 1-99 was present in 122 (28\%), CS 100-399 in 75 (17\%), CS 400-999 in 56 (13\%), and CS $\geq 1000$ in 32 (7\%). MSCTA was normal in 133 (31\%) patients, MSCTA 30-50\% stenosis was observed in 190 (44\%), and MSCTA $\geq 50 \%$ stenosis in 109 (25\%). During follow-up [median 670 days (25th-75th percentile: 418-895)], an event occurred in 21 patients (4.9\%). After multivariate correction for CS, MSCTA $\geq 50 \%$ stenosis, the number of diseased segments, obstructive segments, and non-calcified plaques were independent predictors with an incremental prognostic value to CS.

Conclusion $\quad$ MSCTA provides additional information to CS regarding stenosis severity and plaque composition. This additional information was shown to translate into incremental prognostic value over CS.

Keywords

Imaging • Atherosclerosis • Prognosis

\section{Introduction}

Non-invasive imaging plays an important role in the diagnosis and prognosis of coronary artery disease (CAD). The development of non-invasive anatomic imaging techniques, such as coronary calcium scoring (CS) and multi-slice computed tomography coronary angiography (MSCTA), have resulted in substantially increased interest in non-invasive imaging of atherosclerosis.
Extensive data are available supporting the value of CS in riskstratification and patients with increased CS are generally considered to have a higher likelihood of future cardiac events. The majority of data however have been obtained in asymptomatic patients at low to intermediate risk although the technique may also be useful in symptomatic patients. ${ }^{1-4}$

Direct non-invasive detection of luminal narrowing has become possible with the introduction of MSCTA. Preliminary studies

* Corresponding author. Tel: +3171 5262020, Fax: +3171 5266809, Email: j.j.bax@lumc.nl

Published on behalf of the European Society of Cardiology. All rights reserved. (C) The Author 2009. For permissions please email: journals.permissions@oxfordjournals.org. 
addressing the prognostic value of MSCTA have demonstrated a low risk for events in case of a normal MSCTA study compared with a higher risk in the presence of significant CAD on MSCTA. ${ }^{5-8}$ Importantly, MSCTA is not restricted to luminography and the technique allows simultaneous visualization of the vessel wall. As a result, also non-stenotic lesions can be identified and even some information on plaque composition can be derived. ${ }^{9}$ This may be an important feature of the technique as several plaque characteristics observed on MSCTA have been linked to acute coronary syndromes in retrospective studies. ${ }^{10,11}$ Nevertheless, only limited prospective data are currently available supporting this notion.

Accordingly, both CS and MSCTA may be useful for riskstratification in patients with suspected CAD, it is unclear however if the information regarding stenosis severity and plaque composition on MSCTA may provide additional prognostic information to CS. To answer this clinical question among others, we have started a prospective registry that addresses the prognostic value of MSCTA in relation to baseline characteristics as well as other imaging techniques. ${ }^{12}$ The purpose of the present study was to assess the relationship between observations on CS and MSCTA and to determine whether the information regarding stenosis severity and plaque composition on MSCTA translates into incremental prognostic value over CS alone.

\section{Methods}

\section{Patient selection}

The study population consisted of patients with suspected CAD who were clinically referred for further cardiac assessment because of chest pain, a positive exercise ECG test, or a high-risk profile for cardiovascular disease as part of an ongoing study protocol addressing the prognostic value of MSCTA in relation to other imaging techniques. From this prospective registry, results addressing the incremental prognostic value of MSCTA over myocardial perfusion imaging have been recently published. ${ }^{12}$ Patients were enrolled at the University Hospital in Zurich, Switzerland, and at the Leiden University Medical Center, The Netherlands. The included patients prospectively underwent a CS scan followed by MSCTA. Exclusion criteria were: cardiac arrhythmias, renal insufficiency (defined as a glomerular filtration rate $<30 \mathrm{~mL}$ / $\mathrm{min}$ ), known hypersensitivity to iodine contrast media, and pregnancy. In addition, patients with an uninterpretable MSCTA examination were excluded. The pre-test likelihood of CAD was determined using the Diamond and Forrester method, with a risk threshold of $<13.4 \%$ for low risk, $>87.2 \%$ for high risk, and between 13.4 and $87.2 \%$ for intermediate risk, as previously described. ${ }^{13}$ The study was approved by the Local Ethics Committee of both participating centres.

\section{Calcium scoring and multi-slice computed tomography coronary angiography protocol}

Patients were scanned using a 64-slice CT scanner (Aquillion64, Toshiba Medical Systems, Tokyo, Japan or General Electrics LightSpeed VCT, Milwaukee, WI, USA). Before CS and MSCTA examinations, the patient's heart rate and blood pressure were monitored. In the absence of contraindications, patients with a heart rate exceeding the threshold of 65 b.p.m. were administered beta-blocking medication (50-100 mg metoprolol oral, or 5-10 mg metoprolol i.v.). Before the helical scan, a non-enhanced low-dose ECG-gated scan was performed to measure CS. The CS scan was prospectively triggered at $70 \%$ or
$75 \%$ of the $R-R$ interval and performed using the following scan parameters: $4 \times 3.0 \mathrm{~mm}$ or $2.5 \mathrm{~mm}$; gantry rotation time, $350-500 \mathrm{~ms}$; tube voltage, $120 \mathrm{kV}$; and tube current, $200-250 \mathrm{~mA}$.

The CS scan was used to determine the start and end positions of the MSCTA examination. The helical scan was performed using a collimation of $64 \times 0.5 \mathrm{~mm}$ or $64 \times 0.625 \mathrm{~mm}$. All scan parameters have been previously published. ${ }^{14,15}$

Datasets were reconstructed from the retrospectively gated raw data. Images were reconstructed with an effective slice thickness of 0.3 or 0.5 . Coronary arteries were evaluated using the reconstruction dataset with the least motion artefacts, typically an end-diastolic phase.

The effective dose of the CS and MSCTA scans was estimated from the dose-length product and an organ weighing factor $[k=0.014 \mathrm{mSv} \times$ $\left.(\mathrm{mG} y \times \mathrm{cm})^{-1}\right]$ for the chest as the investigated anatomical region. ${ }^{16}$

\section{Data analysis}

Post-processing of the CS and MSCTA examinations was performed on dedicated workstations (Vitrea2, Vital Images, USA and Advantage, GE Healthcare, USA). The CS was calculated using the Agatston method. ${ }^{17}$ MSCTA angiograms were examined using the axial slices, curved multiplanar reconstructions, and maximum intensity projections. Coronary anatomy was assessed in a standardized manner by dividing the coronary artery tree into 17 segments according to the modified American Heart Association classification. First segments were classified based on the maximum luminal diameter stenosis. Normal MSCTA was defined as completely normal anatomy or minimal wall irregularities $<30 \%$, nonsignificant CAD was defined as the presence of luminal narrowing with a maximal luminal diameter stenosis $<50 \%$, and significant CAD was defined as the presence of an atherosclerotic lesion exceeding the threshold of $50 \%$ maximal luminal diameter stenosis. After assessment of stenosis severity, plaque composition was determined in all diseased segments (non-significant or significant CAD on MSCTA). Plaque composition was graded as non-calcified plaque (plaques having lower density compared with the contrast-enhanced lumen), calcified plaque (plaques with high density), and mixed plaque (containing elements of both non-calcified and calcified plaques).

\section{Follow-up}

Patient follow-up data were gathered using clinical visits or standardized telephone interviews. A composite endpoint was construed using the following events: all-cause mortality, non-fatal myocardial infarction, and unstable angina requiring revascularization. Non-fatal infarction was defined based on the criteria of typical chest pain, elevated cardiac enzyme levels, and typical changes on the ECG. Unstable angina was defined according to the European Society of Cardiology guidelines as acute chest pain with or without the presence of ECG abnormalities, and negative cardiac enzyme levels. ${ }^{18}$ Patients with stable complaints undergoing an early elective revascularization within 60 days after imaging with CS and MSCTA were excluded from the survival analysis.

\section{Statistical analysis}

Continuous variables were expressed as mean and standard deviation, and categorical baseline data were expressed in numbers and percentages. Cox regression analysis was used to determine the prognostic value of CS, and MSCTA variables. First univariate analysis of baseline CS and MSCTA variables was performed using the composite endpoint of all-cause mortality, non-fatal infarction, and unstable angina requiring revascularization. For each variable, a hazard ratio with a $95 \%$ confidence interval $(95 \% \mathrm{Cl})$ was calculated. The predictive value of CS was assessed using binary cut-off values (CS $>0$, CS $\geq 100$, CS $\geq 400$, and CS $\geq 1000$ ). For the survival analysis of MSCTA, plaque 
burden [number of (non) significantly diseased segments] and plaque composition (number of segments with non-calcified, mixed, or calcified plaque) were analysed as continuous variables. Finally, multivariate models were created to assess the independent predictive value of MSCTA corrected for CS and baseline clinical variables. The incremental value of MSCTA over CS and baseline clinical variables was assessed by calculating the global $\chi^{2}$ values.

Statistical analyses were performed using SPSS software (version 12.0, SPSS Inc., Chicago, IL, USA). A $P$-value $<0.05$ was considered statistically significant.

\section{Results}

The current study population, derived from our prospective registry, ${ }^{12}$ consisted of 533 patients presenting with suspected CAD at the University Hospital Zurich $(n=270)$ and at the Leiden University Medical Center $(n=263)$. The demographics in the two populations were similar. In 24 (4.5\%) of these patients, the MSCTA examination was uninterpretable because of the presence of motion artefacts, increased noise owing to a high body mass index, and breathing. In addition, 35 patients (6.9\%) were lost to follow-up, and an early revascularization occurred in 42 (8.3\%) patients. After exclusion of these patients, a total of 432 remained for further analysis. The baseline characteristics of the patient population are presented in Table 1. In summary, the average age of the study cohort was $58 \pm 11$ years and $58 \%$ of patients were men. A low or intermediate pre-test likelihood was observed in, respectively, $24 \%$ and $65 \%$ of patients. A high pre-test likelihood was observed in $11 \%$.

\section{Calcium scoring and multi-slice computed tomography coronary angiography results}

The average CS of the cohort was $290 \pm 730$. Calcium was absent in $117(34 \%)$ patients, a CS of $1-99$ was observed in 122 (28\%), a

\section{Table I Patient characteristics}

\begin{tabular}{|c|c|}
\hline & Total $(n=432)$ \\
\hline Gender (male) & $59 \%$ \\
\hline Age (years) & $58 \pm 11$ \\
\hline \multicolumn{2}{|l|}{ Risk factors } \\
\hline Diabetes & $121(28 \%)$ \\
\hline Hypertension & $244(57 \%)$ \\
\hline Hypercholesterolaemia & $170(39 \%)$ \\
\hline Family history of CAD & 157 (36\%) \\
\hline Current smoking & $119(28 \%)$ \\
\hline Obesity $(\mathrm{BMI} \geq 30)$ & $92(21 \%)$ \\
\hline \multicolumn{2}{|l|}{ Pre-test likelihood of CAD } \\
\hline Low & $102(24 \%)$ \\
\hline Intermediate & $281(65 \%)$ \\
\hline High & $49(11 \%)$ \\
\hline
\end{tabular}

BMI, body mass index; CAD, coronary artery disease.
CS between 100 and 399 in 75 (17\%), a CS between 400 and 999 in 56 (13\%), and a CS $\geq 1000$ was present in 32 (7\%) patients. MSCTA was normal in 133 (31\%) patients, and atherosclerosis (non-significant or significant CAD) was present in the remaining 299 (69\%) patients. Among the patients with atherosclerosis, nonsignificant CAD was observed in 190 (44\%), and significant CAD was present in 109 (25\%) patients. For the GE scanner, the estimated average radiation dose for the MSCTA protocol was $18.3 \pm 5.9 \mathrm{mSv}$, and the average radiation dose for the CS protocol was $1.2 \pm 0.6 \mathrm{mSv}$. For the Toshiba scanner, the estimated average radiation dose for the coronary angiography protocol was $17.6 \pm 5.6 \mathrm{mSv}$ and the estimated radiation dose for the CS protocol was $1.5 \pm 0.7 \mathrm{mSv}$.

\section{Relationship between calcium scoring and stenosis severity and plaque composition assessed on multi-slice computed tomography coronary angiography \\ Stenosis severity}

Figure 1 illustrates the MSCTA findings in patients with increasing CS values. In patients without any coronary calcium, MSCTA was normal in $80 \%$. Non-calcified plaque was observed in the remaining $20 \%$ of patients ( $7 \%$ of the total study population). Importantly, significant CAD was observed in $4 \%$ of patients with a CS of 0 . An example of a patient with a CS of 0 and a large non-calcified plaque on MSCTA, confirmed on conventional coronary angiography is shown in Figure 2. In patients with coronary calcifications, nonsignificant and significant lesions were present in all but a few patients. However, the relationship between CS and significant CAD on MSCTA was less evident. Particularly, in patients with an intermediate CS between 100 and 1000, significant CAD was observed in approximately $50 \%$ of patients. In patients with a high CS $\geq 1000$, non-significant CAD was observed in $30 \%$ of patients.

\section{Plaque composition}

MSCTA enables assessment of plaque composition in addition to stenosis severity. Figure 3 shows the distribution of segments with non-calcified plaque, mixed plaque, and calcified plaque in each CS category as a percentage of the total diseased segments. In patients with CS of 0 , all 76 diseased segments showed noncalcified plaque. With increasing CS categories, the percentage of segments with calcified plaque contributing to the total of diseased segments increased. MSCTA was however able to identify a large proportion of diseased segments with elements of non-calcified plaque (either mixed plaque or non-calcified plaque) in each category.

\section{Follow-up results}

A median follow-up time of 670 days (25th-75th percentile: $418-$ 895) was obtained, during which the composite endpoint occurred in 21 patients (4.9\%). All-cause death was reported in six patients (1.4\%), whereas non-fatal myocardial infarction occurred in eight patients $(1.8 \%)$, and seven patients $(1.6 \%)$ were revascularized owing to unstable angina pectoris. 


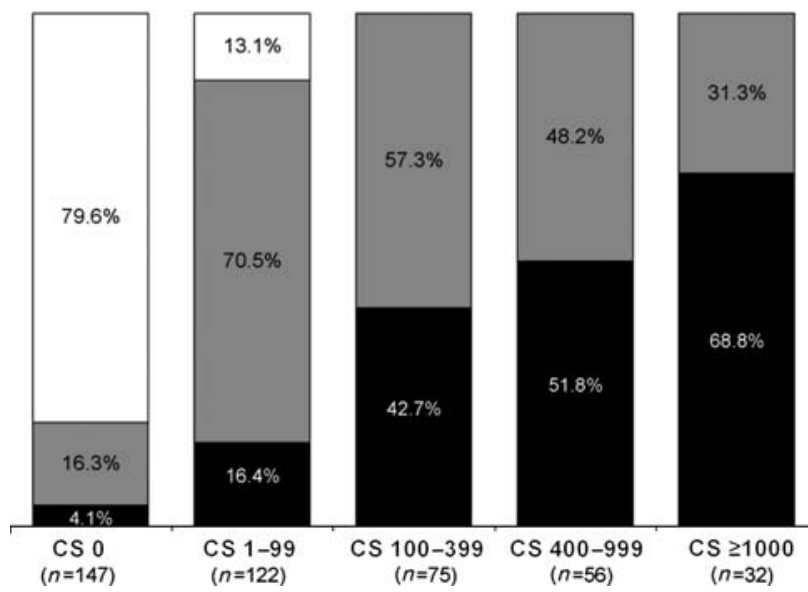

Normal (MSCT normal or minor wall irregularities)

$\square$ Non-significant CAD (MSCT $<50 \%$ stenosis)

- Significant CAD

(MSCT $\geq 50 \%$ stenosis)

Figure I The relationship between increasingly higher calcium scoring categories and the prevalence of normal coronary anatomy (completely normal or minor wall irregularities), non-significant CAD (MSCTA $<50 \%$ stenosis) and significant CAD (MSCTA $\geq 50 \%$ stenosis) on MSCTA. CAD, coronary artery disease; CS, calcium score; MSCTA, multi-slice computed tomography.
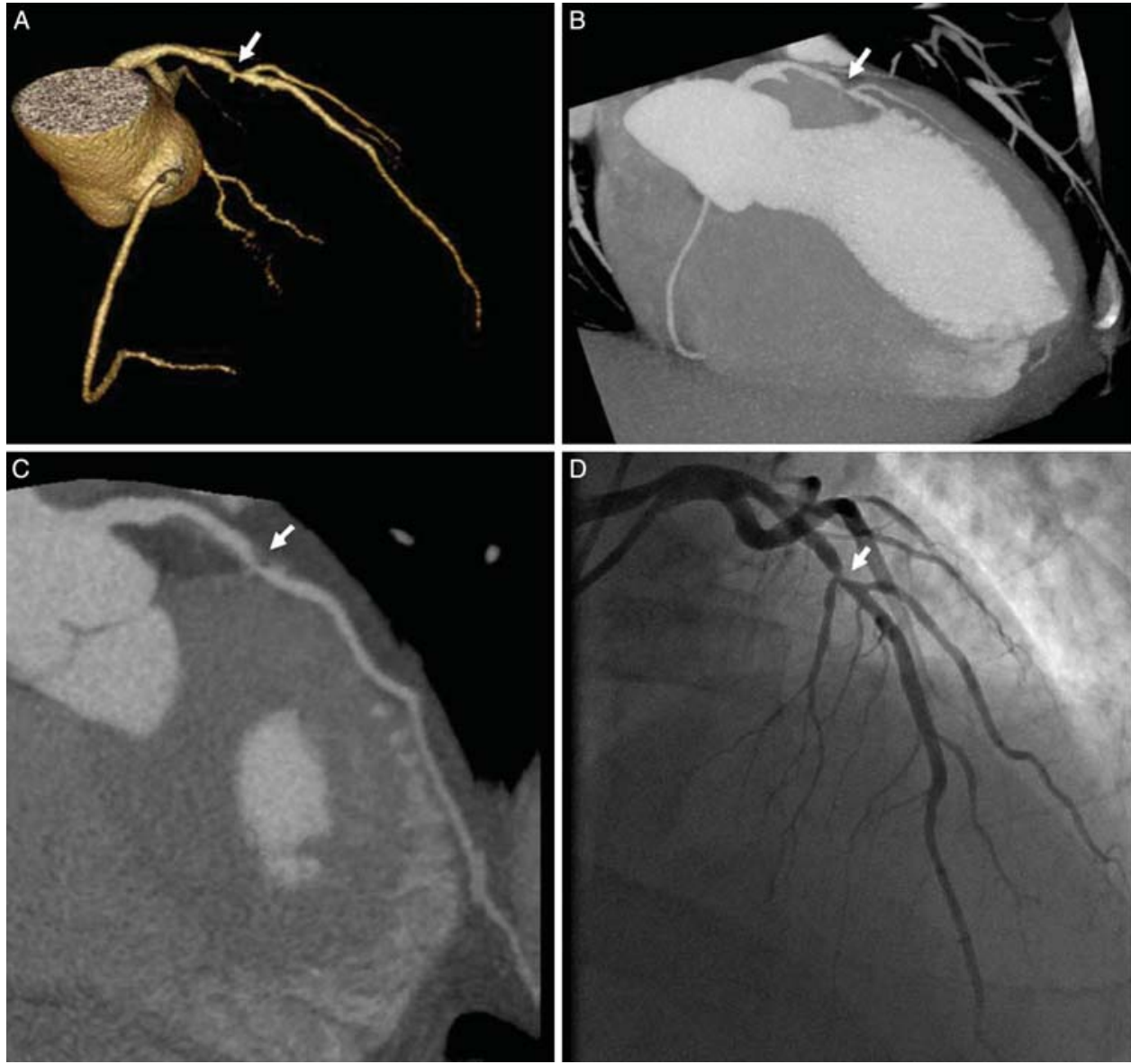

Figure 2 Case example of a 54-year-old male patient presenting with atypical complaints. On CS no coronary calcifications were observed. However, a large significant non-calcified plaque was detected in the left anterior descending artery on MSCTA ( $A-C)$, which was confirmed on conventional coronary angiography $(D)$. CS, calcium score; MSCTA, multi-slice computed tomography. 


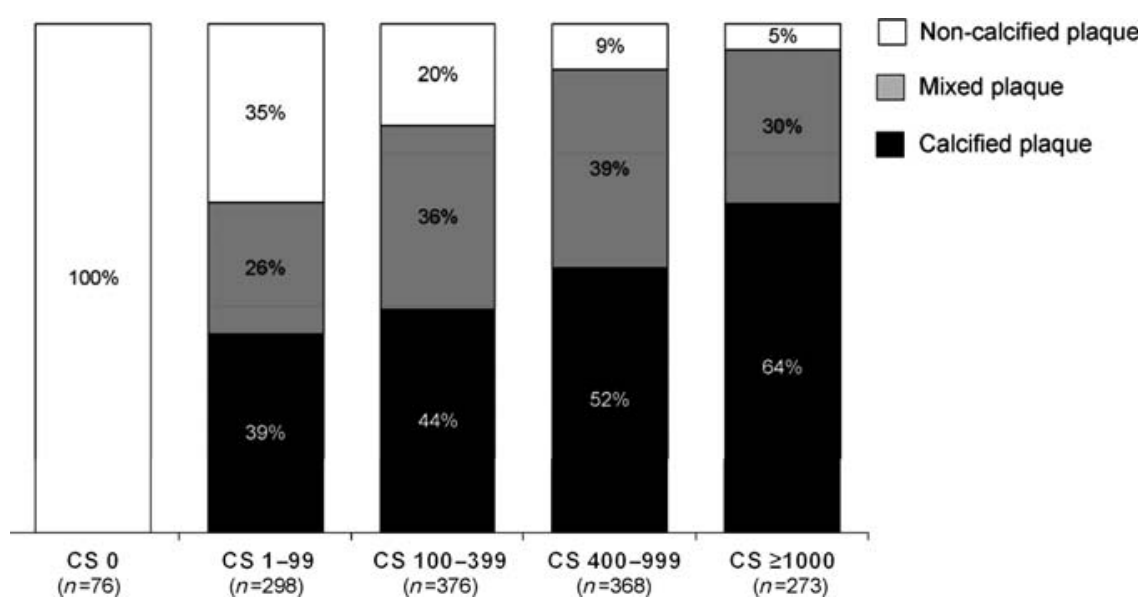

Figure 3 The distribution of segments with non-calcified plaque, mixed plaque, and calcified plaque as a percentage of the total diseased segments on MSCTA per CS category. In patients with a CS of 0 , all 76 diseased segments showed non-calcified plaques. With increasing CS categories, the percentage of diseased segments with calcified plaque increased. Nevertheless, non-calcified plaque and mixed plaque were still observed in a large proportion of diseased segments in each CS category. CS, calcium score; MSCTA, multi-slice computed tomography coronary angiography.

Table 2 Univariate calcium scoring (CS) and multi-slice computed tomography coronary angiography (MSCTA) predictors of coronary events

\begin{tabular}{|c|c|c|}
\hline & HR $(95 \% \mathrm{Cl})$ & $P$-value \\
\hline \multicolumn{3}{|l|}{ CS } \\
\hline $\begin{array}{l}\text { Calcium score (per unit increase } \\
\text { in CS) }\end{array}$ & $1.00(1.00-1.01)$ & 0.019 \\
\hline Any calcium & $3.2(0.9-10.9)$ & 0.062 \\
\hline Calcium score $\geq 100$ & $3.9(1.5-10.2)$ & 0.005 \\
\hline Calcium score $\geq 400$ & $3.5(1.5-8.3)$ & 0.004 \\
\hline Calcium score $\geq 1000$ & $4.1(1.5-11.3)$ & 0.006 \\
\hline \multicolumn{3}{|l|}{ MSCTA } \\
\hline Atherosclerosis & $4.3(1.0-18.6)$ & 0.048 \\
\hline Significant CAD & $3.9(1.7-9.3)$ & 0.002 \\
\hline No. of diseased segments & $1.2(1.08-1.3)$ & $<0.001$ \\
\hline $\begin{array}{l}\text { No. of segments with significant } \\
\text { CAD }\end{array}$ & $1.4(1.2-1.6)$ & $<0.001$ \\
\hline $\begin{array}{l}\text { No. of segments with non-calcified } \\
\text { plaque }\end{array}$ & $1.2(1.07-1.4)$ & 0.003 \\
\hline No. of segments with mixed plaque & $1.3(1.07-1.5)$ & 0.005 \\
\hline $\begin{array}{l}\text { No. of segments with calcified } \\
\text { plaque }\end{array}$ & $1.09(0.9-1.3)$ & 0.254 \\
\hline
\end{tabular}

$\mathrm{CAD}$, coronary artery disease; $\mathrm{HR}$, hazards ratio; $\mathrm{Cl}$, confidence interval.

\section{Survival analysis}

Univariate analysis of CS and MSCTA categories is shown in Table 2. Within the CS cut-off categories CS $\geq 100, C S \geq 400$, and $C S \geq 1000$ were significant predictors of events. The highest hazard ratio was observed when using a cut-off of 1000. On MSCTA several variables reached statistical significance. The presence of any stenosis (non-significant (30-50\% stenosis) or significant ( $\geq 50 \%$ stenosis) as well as the presence of significant CAD ( $\geq 50 \%$ stenosis) were both strong significant predictors. When regarding plaque burden, both the number of diseased segments as well as the number of segments with significant CAD were significant univariate predictors. When regarding plaque composition, the number of segments with non-calcified plaque and the number segments with mixed plaque were also significant predictors of events. The number of segments with calcified plaque was not a significant predictor of events.

\section{Event rates}

The annualized event rate in patients without any coronary calcium was $1.1 \%$. Increasingly higher CS was associated with increasingly higher annualized event rates, the annualized event rate was $1.4 \%$ in patients with a CS $1-99,3.7 \%$ in patients with a CS $100-399$, and $4.8 \%$ in patients with a CS 400-999. The highest annualized event rate of $8.5 \%$ was observed in patients with a CS $\geq 1000$.

When regarding MSCTA, an event rate of $0.8 \%$ was observed in patients with a normal MSCTA (completely normal or minor wall irregularities); while in patients with atherosclerosis (nonsignificant and/or significant CAD) the annualized event rate was 3.5\%. In patients with non-significant CAD, the annualized event rate was $2.1 \%$, vs. $5.9 \%$ in patients with significant CAD.

\section{Independent and incremental prognostic value of multi-slice computed tomography coronary angiography over calcium scoring}

To determine the independent prognostic value of MSCTA, multivariate models were created including all MSCTA variables corrected for age, gender, and $C S \geq 1000$. Table 3 shows that the presence of significant CAD, the number of diseased segments, 
Table 3 Multivariate models for the prediction of coronary events

\begin{tabular}{|c|c|c|}
\hline & HR (95\% Cl) & $P$-value \\
\hline \multicolumn{3}{|l|}{ Model $1 *$} \\
\hline Atherosclerosis & $4.5(0.9-21.3)$ & 0.056 \\
\hline Calcium score $\geq 1000$ & $4.0(1.3-12.4)$ & 0.016 \\
\hline \multicolumn{3}{|l|}{ Model 2* } \\
\hline Significant CAD & $3.6(1.4-9.4)$ & 0.009 \\
\hline Calcium score $\geq 1000$ & $2.9(0.9-9.3)$ & 0.064 \\
\hline \multicolumn{3}{|l|}{ Model 3* } \\
\hline No. of diseased segments & $1.2(1.1-1.3)$ & 0.006 \\
\hline Calcium score $\geq 1000$ & $2.0(0.6-6.9)$ & 0.268 \\
\hline \multicolumn{3}{|l|}{ Model 4* } \\
\hline No. of segments with significant CAD & $1.3(1.1-1.5)$ & 0.003 \\
\hline Calcium score $\geq 1000$ & $2.6(0.7-9.3)$ & 0.148 \\
\hline \multicolumn{3}{|l|}{ Model 5* } \\
\hline $\begin{array}{l}\text { No. of segments with non-calcified } \\
\text { plaque }\end{array}$ & $1.3(1.1-1.4)$ & 0.001 \\
\hline Calcium score $\geq 1000$ & $5.8(1.9-18.2)$ & 0.003 \\
\hline \multicolumn{3}{|l|}{ Model 6* } \\
\hline No. of segments with mixed plaque & $1.2(1.0-1.4)$ & 0.039 \\
\hline Calcium score $\geq 1000$ & $3.6(1.1-11.6)$ & 0.029 \\
\hline
\end{tabular}

obstructive segments, segments with non-calcified plaque, and the number of segments with mixed plaque remained independent predictors. Furthermore, plaque burden and plaque composition provided incremental prognostic value over clinical variables and MSCTA as shown in Figures 4 and 5. These results suggest that MSCTA may provide additional prognostic information. In particular, the number of segments with significant CAD and the number of segments with non-calcified plaque provided significant incremental prognostic value over CS. The number of mixed plaques provided borderline significant $(P=0.058)$ incremental prognostic value to $\mathrm{CS}$.

\section{Discussion}

The main finding of the current study was that MSCTA may provide additional anatomic information regarding stenosis severity and plaque composition compared with CS. Furthermore, this information offers important prognostic information which is incremental to CS for risk-stratification.

\section{Calcium scoring and multi-slice computed tomography coronary angiography for atherosclerosis detection}

In asymptomatic patients, CS may be useful to identify the presence of atherosclerosis and thus identify patients that may be at higher risk than recognized based on traditional risk assessment. ${ }^{19}$

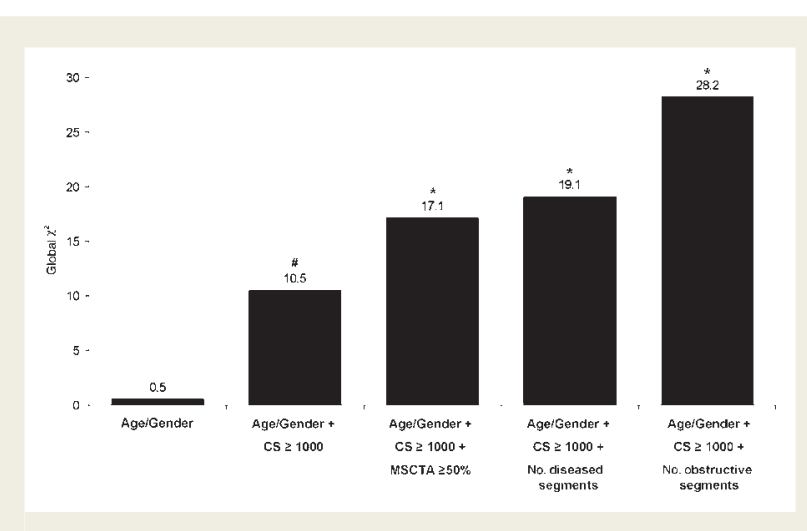

Figure 4 Bar graphs illustrating the incremental prognostic value (depicted by $\chi^{2}$ value on the $y$-axis) of significant CAD ( $\geq 50 \%$ stenosis) on MSCTA and plaque burden (defined as the number of diseased or segments with significant $C A D$ on MSCTA) over age, gender, and CS. CS has a significant incremental prognostic value over age and gender (\#). A further incremental prognostic value over age, gender, and CS was observed with the addition of MSCTA $(*)$. CS, calcium score; MSCTA, multislice computed tomography.

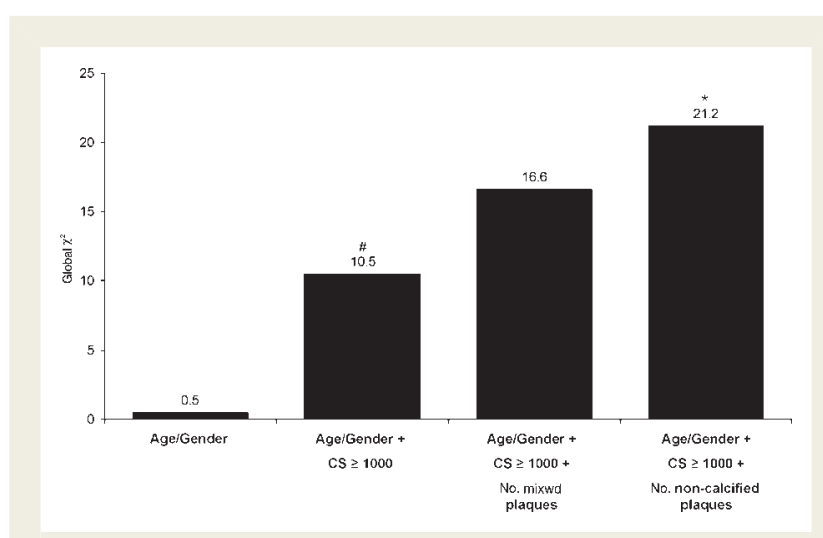

Figure 5 Bar graphs illustrating the incremental prognostic value (depicted by $\chi^{2}$ value on the $y$-axis) of MSCTA plaque composition variables over age, gender, and CS. CS has a significant incremental prognostic value over age and gender (\#). A further incremental prognostic value over age, gender, and CS was observed with the addition of MSCTA $(*)$. CS, calcium score; MSCTA, multi-slice computed tomography.

Vice versa, absence of calcium in general implies a low likelihood of events. ${ }^{20}$ However, in a small proportion of patients with a negative CS, non-calcified plaque has been observed. ${ }^{21,22}$ Indeed, in certain subpopulations, such as those at higher risk or in symptomatic patients, the prevalence of non-calcified plaque may be higher. $^{23-25}$ Akram et al. ${ }^{23}$ specifically studied the relationship between symptomatic status and the prevalence of non-significant and significant CAD in patients with a CS of 0 and observed $22 \%$ non-significant and significant $C A D$ and $8 \%$ significant $C A D$ in symptomatic patients. In the current study, non-significant or significant CAD was identified on MSCTA in $20 \%$ of patients with a CS of 0 , whereas significant CAD was observed in 4\%. Accordingly, 
particularly in symptomatic populations, a small proportion of patients with atherosclerosis may not be recognized by CS.

Considering patients with evidence of coronary calcifications, a positive association has been observed between CS and the presence of significant CAD on MSCTA and conventional coronary angiography. ${ }^{26,27}$ Indeed, in the current study, the prevalence of significant CAD paralleled increasing CS categories. Nevertheless, in a substantial proportion of patients with extensive calcifications, significant CAD was absent on MSCT, indicating somewhat lower specificity of $C S$ to diagnose significant $C A D$ in line with previous investigations. ${ }^{28,29}$ Since particularly in symptomatic patients identification of significant CAD is of importance for guiding clinical management (including decisions for potential revascularization), MSCTA may provide incremental diagnostic information to CS in this regard.

\section{Prognostic value of calcium scoring and multi-slice computed tomography coronary angiography}

A considerable amount of evidence regarding risk-stratification is available with CS in asymptomatic patients. ${ }^{19,20,30-33}$ Although less frequently studied, also in symptomatic patients, CS has been shown to provide important prognostic information. ${ }^{1-4}$ In the study by Detrano et al., ${ }^{1}$ a six times higher event rate was observed in patients with a CS above the median compared to those with a CS below the median.

The prognostic value of MSCTA has been studied less extensively. ${ }^{5-8}$ In the largest study thus far by Min et al., ${ }^{6}$ a cohort of 1127 patients undergoing 16-slice MSCT was evaluated. The prognostic value of the Duke Prognostic Coronary Artery Disease Index was assessed and event rates for all-cause mortality ranging between $0.3 \%$ for none or mild atherosclerosis (stenosis $<50 \%$ ) to $15 \%$ for mild to moderate left main disease were observed in a period of 2 years. Similar findings were reported in smaller studies by Gilard et al. ${ }^{5}$ and Pundziute et al. ${ }^{7}$ Furthermore, recently published results from our current prospective registry have demonstrated an incremental prognostic value of MSCTA over myocardial perfusion imaging using single-photon emission computed tomography. ${ }^{12}$

To our knowledge, the current study is the first in assessing the incremental prognostic value of both stenosis severity and plaque composition on MSCTA over CS. In a previous study however, the incremental value of plaque burden derived by non-invasive coronary angiography with electron beam computed tomography over CS was assessed by Ostrom et al. ${ }^{34}$ The authors showed that plaque burden, defined as the number of non-significantly or significantly diseased vessels, had independent and incremental value in predicting all-cause mortality independent of age, gender, conventional risk factors, and CS. Similar findings were recently reported by Rubinshtein et al. ${ }^{8}$ Accordingly, in combination with our own observations, it appears that non-invasive measures of the extent and severity of stenosis provide incremental prognostic information over CS.

Importantly, assessment of plaque composition may further enhance risk-stratification. However, only limited prospective data are available addressing the potential relationship between plaque composition on MSCTA and outcome. When exploring plaque composition in the current study, the number of segments with non-calcified plaque as well as the number of segments with mixed plaque was shown to be independently associated with increased risk for events. Interestingly, no such relation was observed for the number of segments with calcified plaque. An explanation may be that CS is more accurate at quantifying calcium burden. Furthermore, when regarding only the number of calcified plaques on MSCT, the calcium in mixed plaques is disregarded. Another explanation may be that non-calcified plaque is more important from a prognostic standpoint. Currently, two previous studies have addressed the prognostic value of plaque composition assessed by MSCTA. ${ }^{7,12}$ Pundziute et al. ${ }^{7}$ observed that the number of mixed plaques was a significant predictor when corrected for baseline clinical variables. In addition, we recently showed that plaque composition on MSCTA provides incremental prognostic value over myocardial perfusion imaging. ${ }^{12}$

\section{Limitations}

Even though the diagnostic accuracy of MSCTA is high, uninterpretable images are still encountered in a small percentage of patients owing to motion because of high or irregular heart rates or breathing during the examination. It is however anticipated that the number of uninterpretable studies will continue to decrease with newer generation scanners. Another potential limitation of MSCTA is the use of iodinated contrast media. As a result MSCTA is contraindicated in patients with renal insufficiency or known hypersensitivity to iodine contrast media. Currently, 64-slice MSCTA is still associated with a significantly higher radiation exposure than CS, although the radiation dose of MSCTA will decrease with the use of dedicated dose-reduction techniques that have recently become available. ${ }^{35-38}$

\section{Conclusion}

Non-invasive anatomic imaging using CS and MSCTA is useful for the detection of atherosclerosis; MSCTA however provides additional information to CS regarding stenosis severity and plaque composition. This additional information was shown to translate into incremental value for risk stratification.

\section{Funding}

J.M.v.W. is financially supported by a research grant from the Netherlands Society of Cardiology (Utrecht, the Netherlands). G.P. is financially supported by the training fellowship grant of the European Society of Cardiology (Sophia Antipolis, France) Huygens scholarship. P.A.K. is supported by a grant from the Swiss National Science Foundation (Berne, Switzerland) (SNSF-professorship grant no. PPOOA-114706), and has research grants from GE Healthcare (Milwaukee, WI, USA). J.J.B. has research grants from Medtronic (Tolochenaz, Switzerland), Boston Scientific (Maastricht, the Netherlands), BMS Medical Imaging (N. Billerica, MA, USA), St Jude Medical (Veenendaal, the Netherlands), Biotronik (Berlin, Germany), GE Healthcare (St Giles, UK), and Edwards Lifesciences (Saint-Prex, Switzerland).

Conflict of interest: none declared. 


\section{References}

1. Detrano R, Hsiai T, Wang S, Puentes G, Fallavollita J, Shields P, Stanford W, Wolfkiel C, Georgiou D, Budoff M, Reed J. Prognostic value of coronary calcification and angiographic stenoses in patients undergoing coronary angiography. J Am Coll Cardiol 1996;27:285-290.

2. Georgiou D, Budoff MJ, Kaufer E, Kennedy JM, Lu B, Brundage BH. Screening patients with chest pain in the emergency department using electron beam tomography: a follow-up study. J Am Coll Cardiol 2001;38:105-110.

3. Schenker MP, Dorbala S, Hong EC, Rybicki FJ, Hachamovitch R, Kwong RY, Di Carli MF. Interrelation of coronary calcification, myocardial ischemia, and outcomes in patients with intermediate likelihood of coronary artery disease: a combined positron emission tomography/computed tomography study. Circulation 2008;117:1693-1700.

4. Keelan PC, Bielak LF, Ashai K, Jamjoum LS, Denktas AE, Rumberger JA, Sheedy PF II, Peyser PA, Schwartz RS. Long-term prognostic value of coronary calcification detected by electron-beam computed tomography in patients undergoing coronary angiography. Circulation 2001;104:412-417.

5. Gilard M, Le Gal G, Cornily J, Vinsonneau U, Joret C, Pennec P, Mansourati J, Boschat J. Midterm prognosis of patients with suspected coronary artery disease and normal multislice computed tomographic findings. Arch Intern Med 2007; 165:1686-1689.

6. Min JK, Shaw LJ, Devereux RB, Okin PM, Weinsaft JW, Russo DJ, Lippolis NJ, Berman DS, Callister TQ. Prognostic value of multidetector coronary computed tomographic angiography for prediction of all-cause mortality. J Am Coll Cardiol 2007;50:1161-1170

7. Pundziute G, Schuijf JD, Jukema JW, Boersma E, de RA, van der Wall EE, Bax JJ. Prognostic value of multislice computed tomography coronary angiography in patients with known or suspected coronary artery disease. J Am Coll Cardiol 2007;49:62-70.

8. Rubinshtein R, Halon DA, Gaspar T, Peled N, Lewis BS. Cardiac computed tomographic angiography for risk stratification and prediction of late cardiovascular outcome events in patients with a chest pain syndrome. Int J Cardiol 2008.

9. Schroeder S, Kopp AF, Baumbach A, Meisner C, Kuettner A, Georg C, Ohnesorge B, Herdeg C, Claussen CD, Karsch KR. Noninvasive detection and evaluation of atherosclerotic coronary plaques with multislice computed tomography. J Am Coll Cardiol 2001;37:1430-1435.

10. Hoffmann U, Moselewski F, Nieman K, Jang IK, Ferencik M, Rahman AM, Cury RC, Abbara S, Joneidi-Jafari H, Achenbach S, Brady TJ. Noninvasive assessment of plaque morphology and composition in culprit and stable lesions in acute coronary syndrome and stable lesions in stable angina by multidetector computed tomography. J Am Coll Cardiol 2006;47:1655-1662.

11. Schuijf JD, Beck T, Burgstahler C, Jukema JW, Dirksen MS, de RA, van der Wall EE, Schroeder S, Wijns W, Bax J. Differences in plaque composition and distribution in stable coronary artery disease versus acute coronary syndromes; non-invasive evaluation with multi-slice computed tomography. Acute Card Care 2007;9:48-53.

12. van Werkhoven JM, Schuijf JD, Gaemperli O, Jukema JW, Boersma E, Wijns W, Stolzmann P, Alkadhi H, Valenta I, Stokkel MP, Kroft LJ, de RA, Pundziute G, Scholte A, van der Wall EE, Kaufmann PA, Bax JJ. Prognostic value of multislice computed tomography and gated single-photon emission computed tomography in patients with suspected coronary artery disease. J Am Coll Cardiol 2009;53:623-632.

13. Diamond GA, Forrester JS. Analysis of probability as an aid in the clinical diagnosis of coronary-artery disease. N Engl J Med 1979;300:1350-1358.

14. Schuijf JD, Wijns W, Jukema JW, Atsma DE, de Roos A, Lamb HJ, Stokkel MPM, Dibbets-Schneider P, Decramer I, De Bondt P, van der Wall EE, Vanhoenacker PK, Bax JJ. The relationship between non-invasive coronary angiography with multi-slice computed tomography and myocardial perfusion imaging. J Am Coll Cardiol 2006;48:2508-2514.

15. Gaemperli O, Schepis T, Kalff V, Namdar M, Valenta I, Stefani L, Desbiolles L, Leschka S, Husmann L, Alkadhi H, Kaufmann PA. Validation of a new cardiac image fusion software for three-dimensional integration of myocardial perfusion SPECT and stand-alone 64-slice CT angiography. Eur J Nucl Med Mol Imaging 2007;34:1097-1106.

16. Hausleiter J, Meyer T, Hermann F, Hadamitzky M, Krebs M, Gerber TC, McCollough C, Martinoff S, Kastrati A, Schomig A, Achenbach S. Estimated radiation dose associated with cardiac CT angiography. JAMA 2009;301:500-507.

17. Agatston AS, Janowitz WR, Hildner FJ, Zusmer NR, Viamonte M Jr, Detrano R. Quantification of coronary artery calcium using ultrafast computed tomography. J Am Coll Cardiol 1990;15:827-832.

18. Bassand JP, Hamm CW, Ardissino D, Boersma E, Budaj A, Fernandez-Aviles F, Fox KA, Hasdai D, Ohman EM, Wallentin L, Wijns W. Guidelines for the diagnosis and treatment of non-ST-segment elevation acute coronary syndromes. Eur Heart J 2007;28:1598-1660.

19. Taylor AJ, Bindeman J, Feuerstein I, Cao F, Brazaitis M, O'Malley PG. Coronary calcium independently predicts incident premature coronary heart disease over measured cardiovascular risk factors: mean three-year outcomes in the Prospective Army Coronary Calcium (PACC) project. J Am Coll Cardiol 2005;46:807-814.
20. Budoff MJ, Shaw LJ, Liu ST, Weinstein SR, Mosler TP, Tseng PH, Flores FR, Callister TQ, Raggi P, Berman DS. Long-term prognosis associated with coronary calcification: observations from a registry of 25,253 patients. J Am Coll Cardiol 2007;49:1860-1870.

21. Cheng VY, Lepor NE, Madyoon H, Eshaghian S, Naraghi AL, Shah PK. Presence and severity of noncalcified coronary plaque on 64-slice computed tomographic coronary angiography in patients with zero and low coronary artery calcium. Am J Cardiol 2007;99:1183-1186.

22. Rubinshtein R, Gaspar T, Halon DA, Goldstein J, Peled N, Lewis BS. Prevalence and extent of obstructive coronary artery disease in patients with zero or low calcium score undergoing 64-slice cardiac multidetector computed tomography for evaluation of a chest pain syndrome. Am J Cardiol 2007;99:472-475.

23. Akram K, O'Donnell RE, King S, Superko HR, Agatston A, Voros S. Influence of symptomatic status on the prevalence of obstructive coronary artery disease in patients with zero calcium score. Atherosclerosis 2008;203:533-537.

24. Ramakrishna G, Miller TD, Breen JF, Araoz PA, Hodge DO, Gibbons RJ. Relationship and prognostic value of coronary artery calcification by electron beam computed tomography to stress-induced ischemia by single photon emission computed tomography. Am Heart / 2007;153:807-814.

25. Shemesh J, Apter S, Itzchak Y, Motro M. Coronary calcification compared in patients with acute versus in those with chronic coronary events by using dualsector spiral CT. Radiology 2003;226:483-488.

26. Kennedy J, Shavelle R, Wang S, Budoff M, Detrano RC. Coronary calcium and standard risk factors in symptomatic patients referred for coronary angiography. Am Heart J 1998;135:696-702.

27. Ho JS, FitzGerald SJ, Stolfus LL, Wade WA, Reinhardt DB, Barlow CE, Cannaday JJ. Relation of a coronary artery calcium score higher than 400 to coronary stenoses detected using multidetector computed tomography and to traditional cardiovascular risk factors. Am J Cardiol 2008;101:1444-1447.

28. Knez A, Becker A, Leber A, White C, Becker CR, Reiser MF, Steinbeck G, Boekstegers P. Relation of coronary calcium scores by electron beam tomography to obstructive disease in 2,115 symptomatic patients. Am J Cardiol 2004;93: 1150-1152.

29. Budoff MJ, Georgiou D, Brody A, Agatston AS, Kennedy J, Wolfkiel C, Stanford W, Shields P, Lewis RJ, Janowitz WR, Rich S, Brundage BH. Ultrafast computed tomography as a diagnostic modality in the detection of coronary artery disease: a multicenter study. Circulation 1996;93:898-904.

30. Detrano R, Guerci AD, Carr J], Bild DE, Burke G, Folsom AR, Liu K, Shea S, Szklo M, Bluemke DA, O'Leary DH, Tracy R, Watson K, Wong ND, Kronmal RA. Coronary calcium as a predictor of coronary events in four racial or ethnic groups. N Engl J Med 2008;358:1336-1345.

31. Greenland P, LaBree L, Azen SP, Doherty TM, Detrano RC. Coronary artery calcium score combined with Framingham score for risk prediction in asymptomatic individuals. JAMA 2004;291:210-215.

32. Greenland P, Bonow RO, Brundage BH, Budoff MJ, Eisenberg MJ, Grundy SM, Lauer MS, Post WS, Raggi P, Redberg RF, Rodgers GP, Shaw LJ, Taylor AJ, Weintraub WS. ACCF/AHA 2007 clinical expert consensus document on coronary artery calcium scoring by computed tomography in global cardiovascular risk assessment and in evaluation of patients with chest pain: a report of the American College of Cardiology Foundation Clinical Expert Consensus Task Force (ACCF/ AHA Writing Committee to Update the 2000 Expert Consensus Document on Electron Beam Computed Tomography) developed in collaboration with the Society of Atherosclerosis Imaging and Prevention and the Society of Cardiovascular Computed Tomography. J Am Coll Cardiol 2007;49:378-402.

33. Shaw LJ, Raggi P, Schisterman E, Berman DS, Callister TQ. Prognostic value of cardiac risk factors and coronary artery calcium screening for all-cause mortality. Radiology 2003;228:826-833.

34. Ostrom MP, Gopal A, Ahmadi N, Nasir K, Yang E, Kakadiaris I, Flores F, Mao SS, Budoff MJ. Mortality incidence and the severity of coronary atherosclerosis assessed by computed tomography angiography. J Am Coll Cardiol 2008;52:1335-1343.

35. Hausleiter J, Meyer T, Hadamitzky M, Huber E, Zankl M, Martinoff S, Kastrati A, Schomig A. Radiation dose estimates from cardiac multislice computed tomography in daily practice: impact of different scanning protocols on effective dose estimates. Circulation 2006;113:1305-1310.

36. Hsieh J, Londt J, Vass M, Li J, Tang X, Okerlund D. Step-and-shoot data acquisition and reconstruction for cardiac X-ray computed tomography. Med Phys 2006;33: 4236-4248.

37. Husmann L, Valenta I, Gaemperli O, Adda O, Treyer V, Wyss CA, Veit-Haibach P, Tatsugami F, von Schulthess GK, Kaufmann PA. Feasibility of low-dose coronary CT angiography: first experience with prospective ECG-gating. Eur Heart J 2008;29:191-197.

38. Rybicki FJ, Otero HJ, Steigner ML, Vorobiof G, Nallamshetty L, Mitsouras D, Ersoy H, Mather RT, Judy PF, Cai T, Coyner K, Schultz K, Whitmore AG, Di Carli MF. Initial evaluation of coronary images from 320-detector row computed tomography. Int J Cardiovasc Imaging 2008;24:535-546. 